Check for updates

Cite this: RSC Adv., 2019, 9, 10832

\title{
Enzyme immobilisation on poly-L-lysine-containing calcium phosphate particles for highly sensitive glucose detection $\uparrow$
}

\author{
Suzuka Kojima, (D) ab Fukue Nagata, ${ }^{a}$ Masahiko Inagaki, ${ }^{\text {ab }}$ Shinichi Kugimiya ${ }^{\mathrm{b}}$ \\ and Katsuya Kato iD *a
}

High catalytic activities of enzymes are necessary for enzyme immobilising technology for the development of glucose sensors. The aim of this study is to synthesise two types of poly(L-lysine)-containing calcium phosphate particles (pLys-HAp) and to achieve the immobilisation of glucose oxidase (GOX) on them. The oxidation activity of GOX immobilised on these particles was more than $80 \%$ compared to that of native GOX (considered to be 100\%). Additionally, the relative activity of GOX immobilised on poly- $\varepsilon^{-}$ lysine-containing HAp ( $\varepsilon$-pLys-HAp) remained approximately $70 \%$ after ten cycles. Moreover, glucose detection was able to be performed in the linear range of 4-400 $\mu \mathrm{M}$ using GOX immobilised on pLysHAp composites. In the direct electrochemistry measurement using the cyclic voltammetry (CV) method, a glassy carbon electrode (GCE) modified by $\varepsilon$-pLys-HAp was a good enzyme electrode and can be used for glucose detection with high sensitivity. From these results, poly(L-lysine)-containing HAp composites can be expected to be enzyme immobilisation agents with high stability and biosensors with high sensitivity.

Received 8th March 2019

Accepted 1st April 2019

DOI: 10.1039/c9ra01764j

rsc.li/rsc-advances measuring the glucose concentration in their blood. ${ }^{10,11}$ Insulin dependent diabetic patients need to control their blood sugar levels. Some of these sensors used as disposable sensors are growing. However, the catalytic activities of GOX are hard to stabilise during the repeated recognition of glucose. Therefore, enzyme immobilising technology that can improve the stability by immobilisation enzymes on carriers has been widely used in the present biosensor technologies. ${ }^{12-14}$

Additionally, GOX immobilising technologies ${ }^{15-18}$ have been widely used for other applications instead of blood sugar level sensors. Wang et al. published that the GOX immobilisation can be applied for biofuel cell (BFC) and used as a detector for microRNA-21 (miRNA-21). ${ }^{19}$ The BFC-based self-powered sensors utilised gold nanoparticles with bare paper fibres as bioelectrodes to wire GOX and bilirubin oxidase. The sensitive detection of miRNA-21 reached over a range of $5 \mathrm{fM}$ to $100 \mathrm{pM}$ with a detection limit of $2.7 \mathrm{fM} .{ }^{19}$ Thus, these technologies have explored the range of applications such as other biosensor and biofuel cell. ${ }^{2021}$ It can be said that the catalytic activities of GOX become important for electrical output power and sensor sensitivity.

Hydroxyapatite $\left(\mathrm{Ca}_{10}\left(\mathrm{PO}_{4}\right)_{6}(\mathrm{OH})_{2}\right.$, HAp) is well known as a type of a calcium phosphate compound with strong affinity for various proteins. It has been frequently reported that HAp has been used as an enzyme immobilising agent. ${ }^{22}$ Bharath et al. prepared a glucose sensor by immobilisation of GOX on reduced graphene oxide sheets with HAp nanorods modified GCE, and these sensor indicated high sensitivity and stability by amperometric glucose determination. ${ }^{23}$ In our previous study, 
we reported that peptide-containing calcium phosphate particles (peptide-HAp) can selectively adsorb proteins. ${ }^{24}$ The selective adsorption of proteins was the result of electrostatic interactions between peptide-HAp and the protein, and these materials were expected to work as carrier materials for proteins. ${ }^{24}$ Furthermore, the high adsorption ability of avidin on poly $(\alpha$-glutamic acid) or poly $(\gamma$-glutamic acid) containing HAp (pGlu-HAp) and its efficient binding activity to biotin were also reported. ${ }^{25}$ It revealed that the morphologies of two pGlu-HAp materials showed plate-like or sheet-like particles due to the effects of peptide structure. The higher maximum adsorption amount for avidin on $\alpha$-pGlu-HAp was due to the difference in the density of carboxyl groups in pGlu-HAp. In conclusion, it revealed that peptide-HAp is a useful composite for development of a biosensor.

Many researchers have reported the synthesis of silica materials in the presence of poly-L-lysine as a template. ${ }^{26-29}$ However, few researchers have reported the calcium phosphate fabricated using poly-L-lysine. Bradley et al. prepared the hydroxyapatite composited with polysaccharides (such as chondroitin sulfate and polygalacturonic acid) or poly amino acid (like poly-L-asparagine and poly-L-lysine). ${ }^{30}$ Gao et al. reported that poly- $\varepsilon$-lysine was introduced to the surface of bacterial cellulose nanofibers via crosslinking for bone-like apatite deposition, and its nanocomposites were expected to be adapted for applications in bone tissue engineering. ${ }^{31}$ Ding et al. synthesised poly-L-lysine/HAp/carbon nanotube hybrid biocomposite scaffolds for antibody immobilisation. ${ }^{32}$ Thus, it can be considered that these biopolymer-hydroxyapatite composites, especially poly- $\varepsilon$-lysine, can find wide applications.

Herein, we report that two types of poly-L-lysine (poly- $\alpha$-lysine and poly-e-lysine) were compounded with HAp to form new poly-lysine containing HAp (pLys-HAp) particles. The aim of this study was to prepare pLys-HAp composites as an enzyme immobilising agent and to immobilise large quantities of GOX with high catalytic activity and high stability. Moreover, we examined the effect of peptides having different structures for these materials and the adsorption properties. The morphologies of the as-synthesised particles were observed, and the oxidative activities were analysed by GOX immobilised on the surface of the samples. Furthermore, we investigated the application as a glucose sensor by immobilised GOX on a glassy carbon electrode (GCE).

\section{Experimental}

\subsection{Materials}

All chemicals were of analytical grade and used without further purification. $\varepsilon$-Polylysine ( $\varepsilon$-pLys) with molecular weight $\left(M_{\mathrm{w}}\right)$ of 4000 was received from JNC CO., Tokyo, Japan. Poly(L-lysine hydrobromide) ( $\alpha$-pLys; $M_{\mathrm{w}}>12000$ ) was purchased from Peptide Institute, Inc. (Osaka, Japan). Calcium acetate monohydrate $\left[\left(\mathrm{CH}_{3} \mathrm{COO}\right)_{2} \mathrm{Ca} \cdot \mathrm{H}_{2} \mathrm{O}\right]$, diammonium hydrogen phosphate $\left[\left(\mathrm{NH}_{4}\right)_{2} \mathrm{HPO}_{4}\right]$, Dulbecco's PBS $(-), \mathrm{D}(+)$-glucose, ethanol, 4-aminoantipyrine (4-AAP) and phenol crystals were obtained from Wako Pure Chemical Industries (Osaka, Japan). Glucose oxidase (GOX) from Aspergillus niger (isoelectric point $(\mathrm{pI})=4.2$,
$\left.M_{\mathrm{w}}=160000 \mathrm{Da}\right)$, graphene oxide, peroxidase from horseradish (POD, $\mathrm{pI}=7.2, M_{\mathrm{w}}=40200$ Da), 8-anilino-1naphthalenesulfonic acid (ANS) and Rhodamine B isothiocyanate (Rhodamine B) were purchased from Sigma-Aldrich Co. (St. Louis., MO, USA). The Bio-Rad protein assay dye reagent concentrate was obtained from Bio-Rad Laboratories (Hercules, CA, USA).

\subsection{Preparation of pLys-HAp particles}

Poly-L-lysine-containing HAp (pLys-HAp) was synthesised as follows: $\operatorname{poly}(\alpha$-lysine) or poly( $\varepsilon$-lysine) $(20,30$ or $40 \mathrm{mg}$ ) was mixed with $200 \mathrm{~mL}\left(\mathrm{NH}_{4}\right)_{2} \mathrm{HPO}_{4}$ solution $(9 \mathrm{mM})$. The solution was further stirred for $30 \mathrm{~min}$ at $20^{\circ} \mathrm{C}$. The mixture was added to a $200 \mathrm{~mL}\left(\mathrm{CH}_{3} \mathrm{COO}\right)_{2} \mathrm{Ca}$ solution $(15 \mathrm{mM})$ and heated to $60{ }^{\circ} \mathrm{C}$ (heating rate of $1{ }^{\circ} \mathrm{C} \min ^{-1}$ ), before it was kept for $3 \mathrm{~h}$ at the same temperature. The solid materials were separated by centrifugation at $6000 \mathrm{rpm}$ for $10 \mathrm{~min}$ and washed with deionised water twice. The final products were obtained via a freeze-drying process.

\subsection{Characterisation of synthesised pLys-HAp}

Field-emission scanning electron microscopy (FE-SEM, S-4300, Hitachi Ltd., Tokyo, Japan) was used to investigate the morphologies of samples at an acceleration voltage of $10.0 \mathrm{kV}$. Transmission electron microscopy (TEM) images were taken using a JEM-2010 (JEOL Ltd., Tokyo, Japan) at an accelerator voltage of $200 \mathrm{kV}$. TriStar 3000 (Shimadzu Co., Kyoto, Japan) was used to measure the nitrogen $\left(\mathrm{N}_{2}\right)$ adsorption-desorption isotherms. The specific surface area, pore volume and pore size distribution were calculated via the Brunauer-Emmett-Teller (BET) and the Barrett-Joyner-Halenda (BJH) methods. Inductively coupled plasma optical emission spectrometry (ICP-OES, IRIS Advantage, Thermo Fisher Scientific Inc., Waltham, MA, USA) was used to determine the $\mathrm{Ca} / \mathrm{P}$ molar ratio of pLys-HAp. Powder X-ray diffraction spectra were obtained using a SmartLab SE/B1 (Rigaku Co., Tokyo, Japan) with $\mathrm{CuK} \alpha$ radiation generated at $40 \mathrm{kV}$ and $30 \mathrm{~mA}$. The scanning speed was $2.0^{\circ} \mathrm{min}^{-1}$ and the $2 \theta$ range varied from $3.0^{\circ}$ to $60.0^{\circ}$. Fouriertransform Infra-Red (FT-IR) spectra were measured to confirm the presence of HAp and peptide in pLys-HAp using FT/IR-4700 spectrometer (JASCO Co., Tokyo, Japan) in the range of 400$4000 \mathrm{~cm}^{-1}$. The contents of calcium, nitrogen and phosphorus of pLys-HAp were analysed using a scanning transmission electron microscopy (STEM, JEM-2100 Plus instrument operated at $200 \mathrm{kV}$, JEOL Ltd., Tokyo, Japan) and an energy dispersive X-ray spectroscopy (EDX, Noran System 7, Thermo Fisher Scientific Inc., Waltham, MA, USA) analysis. To determine the relative amount of peptide in the composites, thermogravimetry and differential thermal analysis (TG-DTA) was performed with a Thermo Plus TG 8120 (Rigaku Co., Tokyo, Japan). The reaction temperature was increased from room temperature to $1000{ }^{\circ} \mathrm{C}$ at a heating rate of $10{ }^{\circ} \mathrm{C} \mathrm{min}^{-1}$. The zeta-potential of pLys-HAp was obtained using a zeta-potential analyser (ELSZ-1000, Otsuka Electronics Co., Tokyo, Japan). The particles were prepared by dispersion in $10 \mathrm{mM}$ phosphate buffer $(\mathrm{pH}=7.0)$ and sonication for $3 \mathrm{~min}$. 


\subsection{GOX adsorption on pLys-HAp}

First, the initial GOX solution was prepared to set the concentration to $500 \mu \mathrm{g} \mathrm{mL}{ }^{-1}$. Each $5 \mathrm{mg}$ of pLys-HAp was added to different amounts of GOX solution (1-3 mL) and stirred overnight at $20{ }^{\circ} \mathrm{C}$. The supernatant was collected by centrifugation at $6000 \mathrm{rpm}$ for $10 \mathrm{~min}$. The excess GOX amounts in the supernatant were calculated according to the Bradford method using Bio-Rad protein assay via UV-vis spectroscopy (Infinite F200 PRO, Tecan Group Ltd., Männedorf, Switzerland) at $\lambda=$ $595 \mathrm{~nm}$. The adsorption amounts of GOX was determined by subtracting the excess amounts of GOX from the initial GOX amounts using the following equation: ${ }^{33}$

$$
Q_{\mathrm{e}}=Q\left(\frac{I-I_{1}}{I}\right)
$$

where $Q_{\mathrm{e}}$ is GOX adsorption capacity on pLys-HAp and $Q$ is initial GOX amount. $I$ and $I_{1}$ are absorbance intensities in the supernatant before and after adsorption.

\subsection{The amino group density in pLys-HAp}

The density of amino group in pLys-HAp samples to be used by enzyme adsorption was analysed. Each pLys-HAp materials (1 $\mathrm{mg}$ ) were mixed with $1 \mathrm{~mL}$ of Rhodamine B solution (40 $\mu \mathrm{g}$ $\left.\mathrm{mL}^{-1}\right)$. After stirring overnight at $20{ }^{\circ} \mathrm{C}$ in the dark, the precipitant was separated by centrifugation at $14000 \mathrm{rpm}$ for $5 \mathrm{~min}$. The solid materials were washed four times with $10 \mathrm{mM}$ phosphate buffer at $\mathrm{pH} 7.0$ and resuspended in $10 \mathrm{mM}$ phosphate buffer $(1 \mathrm{~mL})$. The amino group density in the suspension was determined using a spectrofluorophotometer RF-5300PC (Shimadzu Co., Kyoto, Japan). The excitation and emission wavelengths were set as 555 and $580 \mathrm{~nm}$, respectively.

\subsection{Conformational changes in immobilised GOX on pLys- HAp}

The changes of the three-dimensional structures of GOX by the adsorption on pLys-HAp samples were confirmed by ANS method. ${ }^{34-37}$ GOX $(500 \mu \mathrm{g})$ was immobilised on $\alpha$-pLys-HAp (40 $\mathrm{mg}$ ) and $\varepsilon$-pLys-HAp (40 mg) during the overnight stirring at $20{ }^{\circ} \mathrm{C}$. Furthermore, ANS solution $\left(50 \mu \mathrm{g} \mathrm{mL}^{-1}\right.$ in $10 \mathrm{mM}$ phosphate buffer at $\mathrm{pH}$ 7.0) was added to the suspension. The mixture was incubated at $20{ }^{\circ} \mathrm{C}$ for $3 \mathrm{~h}$ in the dark. Finally, the fluorescence spectra were obtained by spectrofluorophotometer (the excitation wavelengths: $387 \mathrm{~nm}$, the fluorescence wavelengths: 400-600 $\mathrm{nm}$ ).

\subsection{The oxidative activity of GOX on pLys-HAp and enzyme kinetics}

GOX was dissolved in $10 \mathrm{mM}$ phosphate buffer at $\mathrm{pH} 7.0$ and the concentration was set to $30 \mu \mathrm{g} \mathrm{mL}{ }^{-1}$. Other solutions such as $4 \mathrm{mM}$ glucose, $1.76 \%$ 4-AAP, $6 \%$ phenol and $1 \mathrm{mg} \mathrm{mL}^{-1}$ POD solution were prepared using the same buffer. Each pLys-HAp sample ( $1 \mathrm{mg}$ ) was added to $1 \mathrm{~mL}$ of GOX solution and the mixture was stirred overnight at $4{ }^{\circ} \mathrm{C}$. After removing the supernatant by centrifugation at $14000 \mathrm{rpm}$ for $5 \mathrm{~min}, 80 \mu \mathrm{L}$ of $4 \mathrm{mM}$ glucose and $850 \mu \mathrm{L}$ of $10 \mathrm{mM}$ phosphate buffer were mixed with the solid material. The mixture was stirred at $25{ }^{\circ} \mathrm{C}$ for $3 \mathrm{~min}$, and $20 \mu \mathrm{L}$ of $1.76 \%$ 4-AAP, $20 \mu \mathrm{L}$ of $6 \%$ phenol and 30 $\mu \mathrm{L}$ of $1 \mathrm{mg} \mathrm{mL}^{-1}$ POD solution were added to the mixture. The mixture was incubated at $25{ }^{\circ} \mathrm{C}$ for $3 \mathrm{~min}$ and the supernatant was separated by centrifugation at $14000 \mathrm{rpm}$ for $3 \mathrm{~min}$. The absorbance of product in the supernatant at $500 \mathrm{~nm}$ was analysed with UV-vis spectrophotometer DU 800 (Beckman Coulter Inc., IN, USA).

The enzyme catalytic kinetics were measured with different concentrations of 4-AAP and calculated from the LineweaverBurk equation: ${ }^{38-41}$

$$
\frac{1}{V}=\frac{K_{\mathrm{m}}}{V_{\max }[\mathrm{S}]}+\frac{1}{V_{\max }}
$$

where $V$ is initial velocity, $K_{\mathrm{m}}$ is Michaelis constant, $V_{\max }$ is the maximum velocity and $[\mathrm{S}]$ is concentration of 4-AAP. The turn over number $K_{\text {cat }}$ and the specificity constant $K_{\text {cat }} / K_{\mathrm{m}}$ were calculated based on this equation.

\subsection{Electrochemical measurement}

The direct electrochemistry of the sample modified glassy carbon electrode (GCE) was investigated by cyclic voltammetry (CV) method using automatic polarisation system HSV-110 (HOKUTO DENKO Co., Tokyo, Japan). The electrochemical measurement was carried out in a three-electrode cell consisting of the GCE, platinum wire as the counter electrode and Ag/ $\mathrm{AgCl}$ saturated $\mathrm{KCl}$ as the reference electrode. The GCE was polished to obtain the mirror-like surface by alumina-in-water slurry. After cleaning with deionised water and ethanol, the electrode was dried at $25{ }^{\circ} \mathrm{C}$.

Each pLys-HAp ( $1 \mathrm{mg}$ ) was mixed with $1 \mathrm{~mL}$ of GOX solution $\left(150 \mu \mathrm{g} \mathrm{mL}^{-1}\right.$ in $10 \mathrm{mM}$ phosphate buffer at $\mathrm{pH}$ 7.0) and stirred overnight at $20{ }^{\circ} \mathrm{C}$. After removing the supernatant from the mixture by centrifugation at $14000 \mathrm{rpm}$ for $5 \mathrm{~min}$, the solid material was redispersed in $100 \mu \mathrm{L}$ of the same buffer by sonication for $3 \mathrm{~min}$. The GOX/pLys-HAp mixture $(20 \mu \mathrm{L})$ was added to $20 \mu \mathrm{L}$ of aluminium silicate nanotube hydrogels (imogolite) ${ }^{42}$ and $20 \mu \mathrm{L}$ of graphene oxide, and the suspension $(20 \mu \mathrm{L})$ was dropped on GCE. After the electrode was dried for $45 \mathrm{~min}$ at $25{ }^{\circ} \mathrm{C}$, the measurements were performed in $\mathrm{O}_{2}$-saturated Dulbecco's PBS ( $\mathrm{pH} 7.3)$ at a scan rate of $100 \mathrm{mV} \mathrm{s}^{-1}$ with the addition of various concentrations of glucose.

\section{Results and discussion}

\subsection{The morphologies of pLys-HAp and their characterisation}

The morphologies of the poly-lysine-containing HAp composites were analysed by FE-SEM and TEM. The particles having round tips were observed and their particle sizes were $\sim 60 \mathrm{~nm}$ as shown in Fig. 1(a-f). However, there were no significant changes in the morphologies of pLys-HAp in comparison with pure HAp by increasing the amount of peptides. It indicated that quite few peptides were included in these materials and the particle morphologies were not affected by the presence of polylysine. 

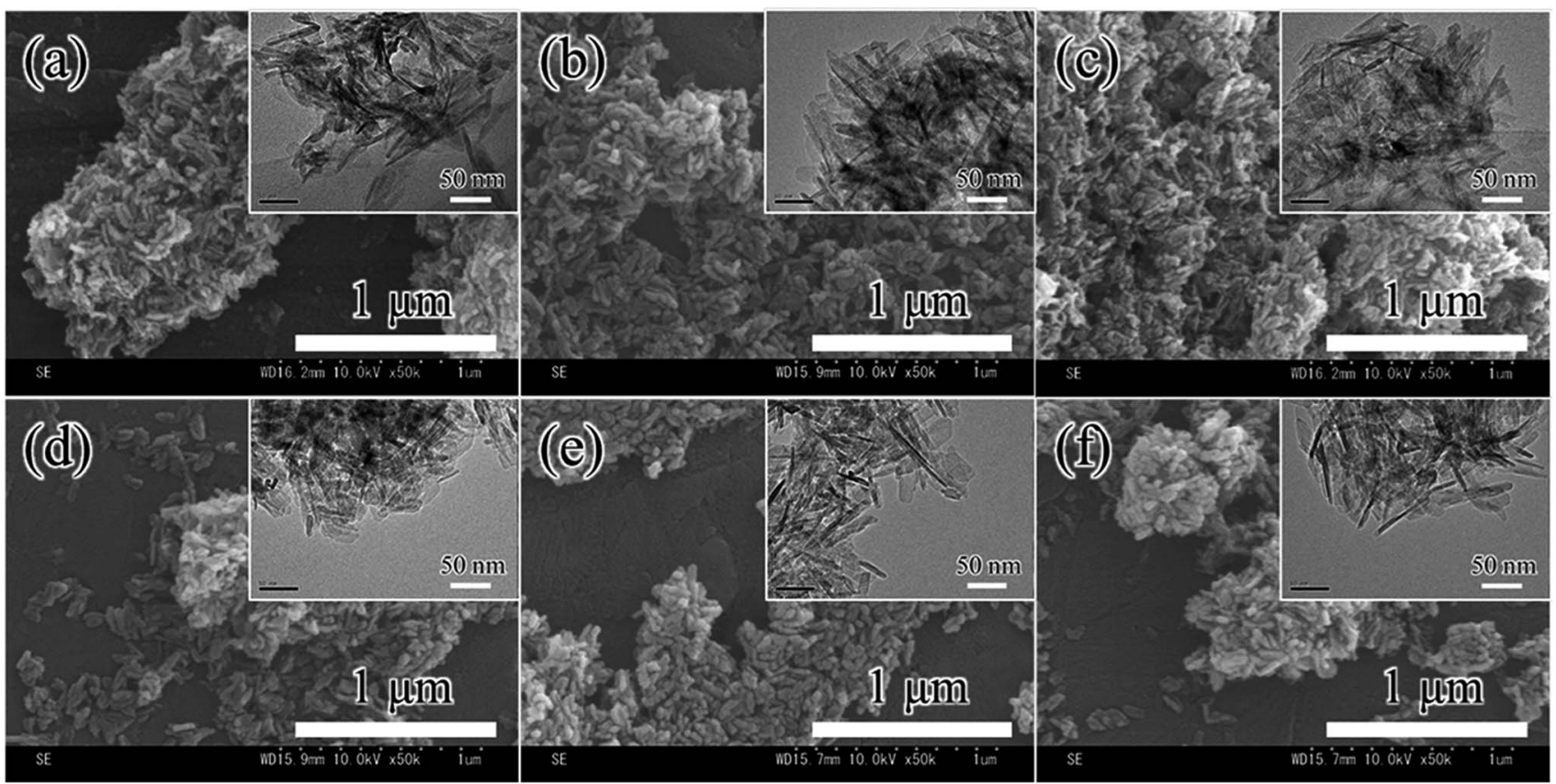

Fig. 1 FE-SEM images of pLys-HAp composites: (a) $\alpha$-pLys-HAp (20 mg), (b) $\alpha$-pLys-HAp (30 mg), (c) $\alpha$-pLys-HAp (40 mg), (d) $\varepsilon$-pLys-HAp (20 $\mathrm{mg})$, (e) $\varepsilon$-pLys-HAp (30 mg) and (f) $\varepsilon$-pLys-HAp (40 mg). The insets in (a-f) show TEM images of each sample.

Table 1 summarises the surface area and the pore volume of pLys-HAp. For $\alpha$-pLys-HAp (20, 30 and $40 \mathrm{mg}$ ) and $\varepsilon$-pLys-HAp $(20,30$ and $40 \mathrm{mg})$, the specific surface areas were 132, 145, $133,148,146$ and $152 \mathrm{~m}^{2} \mathrm{~g}^{-1}$, respectively. The pore volumes of $\alpha$-pLys-HAp (20, 30 and $40 \mathrm{mg})$ and $\varepsilon$-pLys-HAp (20, 30 and 40 $\mathrm{mg}$ ) were $0.80,0.93,0.88,0.98,0.98$ and $1.0 \mathrm{~cm}^{3} \mathrm{~g}^{-1}$, respectively. Moreover, the pore size distribution and the nitrogen adsorption-desorption isotherms of all pLys-HAp samples indicated type IV, as shown in Fig. S1. $\dagger$ Fig. S1(A) $\dagger$ indicates that the pores with a diameter of $30 \mathrm{~nm}$ were only in $\alpha$-pLys-HAp (30 and $40 \mathrm{mg}$ ). Such pore sizes of $30 \mathrm{~nm}$ were appeared by increasing the amount of peptides.

Table 1 shows the $\mathrm{Ca} / \mathrm{P}$ molar ratio of pLys-HAp measured by ICP-OES. The $\mathrm{Ca} / \mathrm{P}$ ratios were $1.45,1.45,1.43,1.43,1.43$ and 1.42 for $\alpha$-pLys-HAp (20,30 and $40 \mathrm{mg})$ and $\varepsilon$-pLys-HAp (20, 30 and $40 \mathrm{mg}$ ), respectively. The values are lower than 1.67 (the stoichiometric ratio of HAp). From these results, it could be considered that the calcium phosphate synthesised in this study were calcium-deficient HAp or low-crystallinity HAp due to HAp and peptides composites.

The diffraction patterns of pLys-HAp composites are shown in Fig. 2(A). The characteristic reflections of all pLys-HAp at $2 \theta=$ $26.0^{\circ}, 31.8^{\circ}, 32.2^{\circ}, 32.8^{\circ}, 34.0^{\circ}, 39.8^{\circ}, 46.6^{\circ}, 49.5^{\circ}$ and $53.3^{\circ}$ are indexed to the (002), (211), (112), (300), (202), (310), (222), (213) and (004) planes of HAp, respectively. All the broad peaks in the synthesised pLys-HAp are in good agreement with the Joint Committee on Powder Diffraction Standards (JCPDS) card (090432) for HAp. The formation of low-crystallinity HAp, thus, was indicated. ${ }^{43-45}$

The FT-IR spectra of $\alpha$-pLys, $\alpha$-pLys-HAp (20, 30 and $40 \mathrm{mg}$ ), $\varepsilon$-pLys and $\varepsilon$-pLys-HAp (20, 30 and $40 \mathrm{mg}$ ) are shown in Fig. 2(B). The two strong adsorption bands observed at around 1020 and $960 \mathrm{~cm}^{-1}$ are attributed to symmetric and asymmetric bending modes of $\mathrm{P}-\mathrm{O}$ in $\mathrm{PO}_{4}{ }^{3-}$ in $\mathrm{HAp}$, respectively. Another two

Table 1 Structural properties, Ca/P molar ratios, amounts of peptide and zeta-potential of pLys-HAp particles

\begin{tabular}{llllll}
\hline Sample & Surface area $^{a}\left(\mathrm{~m}^{2} \mathrm{~g}^{-1}\right)$ & Pore volume $^{a}\left(\mathrm{~cm}^{3} \mathrm{~g}^{-1}\right)$ & Ca/P molar ratio & Amount of peptide $^{c}(\mathrm{mg})$ & Zeta-potential $^{d}(\mathrm{mV})$ \\
\hline$\alpha$-pLys-HAp (20 mg) & 132 & 0.80 & 1.45 & 11 & +13.9 \\
$\alpha$-pLys-HAp (30 mg) & 145 & 0.93 & 1.45 & 11 & +15.4 \\
$\alpha$-pLys-HAp (40 mg) & 133 & 0.88 & 1.43 & 13 & +14.0 \\
E-pLys-HAp (20 mg) & 148 & 0.98 & 1.43 & 5.5 & +4.2 \\
E-pLys-HAp (30 mg) & 146 & 0.98 & 1.43 & 4.1 & +2.8 \\
E-pLys-HAp (40 mg) & 152 & 1.0 & 1.42 & 4.3 & +4.2
\end{tabular}

${ }^{a}$ The specific surface area and pore volume were calculated from the nitrogen adsorption-desorption isotherms via the Brunauer-Emmett-Teller (BET) and the Barrett-Joyner-Halenda (BJH) methods. ${ }^{b}$ The Ca/P molar ratio was determined using an inductively coupled plasma optical emission spectrometry. ${ }^{c}$ The relative amount of peptides in pLys-HAp composites was revealed by thermogravimetry. ${ }^{d}$ The zeta-potential of composites was obtained using the electrophoretic light scattering method. The particles were prepared by dispersion in $10 \mathrm{mM}$ phosphate buffer (pH 7.0) with sonication for $3 \mathrm{~min}$. 
(A)

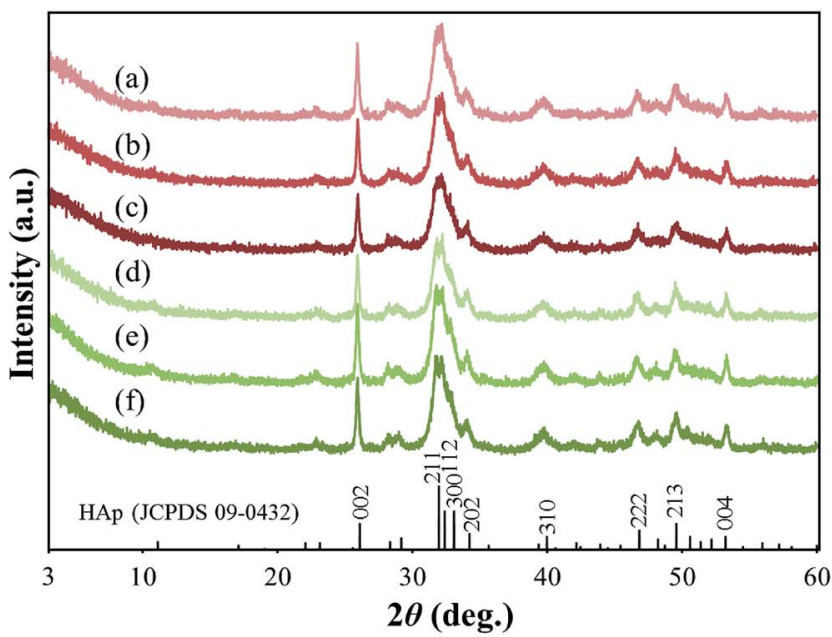

(B)

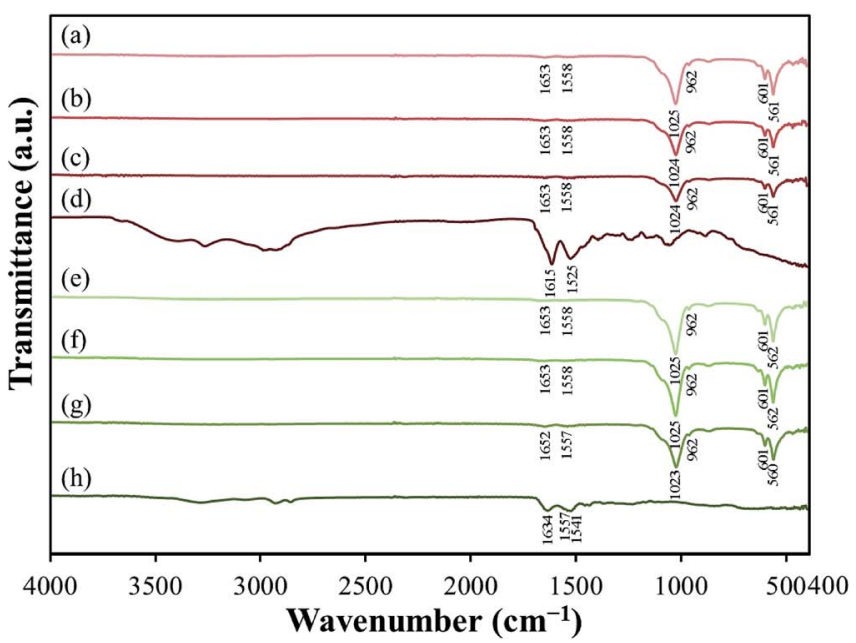

Fig. 2 (A) X-ray diffraction patterns of (a) $\alpha$-pLys-HAp (20 mg), (b) $\alpha$-pLys-HAp (30 mg), (c) $\alpha$-pLys-HAp (40 mg), (d) $\varepsilon$-pLys-HAp (20 mg), (e) $\varepsilon$ pLys-HAp (30 mg) and (f) $\varepsilon$-pLys-HAp (40 mg), respectively (JCPDS card no. 09-0432). (B) FT-IR spectra of (a) $\alpha$-pLys-HAp (20 mg), (b) $\alpha$-pLysHAp (30 mg), (c) $\alpha$-pLys-HAp (40 mg), (d) pure $\alpha$-pLys, (e) $\varepsilon$-pLys-HAp (20 mg), (f) $\varepsilon$-pLys-HAp (30 mg), (g) $\varepsilon$-pLys-HAp (40 mg) and (h) pure $\varepsilon$ pLys, respectively.

stretching bands at 600 and $560 \mathrm{~cm}^{-1}$ are assigned to $\mathrm{O}-\mathrm{P}-\mathrm{O}$ in $\mathrm{PO}_{4}{ }^{3-}$ in HAp, respectively. ${ }^{43-46}$ The stretching vibration of $\mathrm{PO}_{4}{ }^{3-}$ of HAp in all pLys-HAp were observed at around 1024, 962, 601 and $561 \mathrm{~cm}^{-1}$. For pLys, the characteristic peaks appearing from 1550 to $1485 \mathrm{~cm}^{-1}$ and from 1590 to $1660 \mathrm{~cm}^{-1}$ are the symmetric and asymmetric stretches of $-\mathrm{NH}_{2} \cdots \mathrm{H}^{+}$of peptide. ${ }^{4-51}$ In the pure $\alpha$-pLys spectrum, the adsorption bands at $1615 / 1525 \mathrm{~cm}^{-1}$ are attributed to asymmetrical/symmetrical stretching vibrations of $-\mathrm{NH}_{2} \cdots \mathrm{H}^{+}$, respectively. The bands of $-\mathrm{NH}_{2} \cdots \mathrm{H}^{+}$in $\varepsilon$-pLys occur at 1634,1557 and $1541 \mathrm{~cm}^{-1}$. The peaks at $1485-1660 \mathrm{~cm}^{-1}$ for pLys-HAp samples were shifted to the high-wavelength side due to the combination of hydroxyapatite and peptide. These results confirmed that the assynthesised particles consisted of HAp and pLys. Moreover, it can be considered that these pLys-HAp composed from a little amount of peptide because these peaks were not significantly changed by increasing the addition amount of peptides.

The secondary structural contents of two pLys (pure and bound with $\mathrm{PO}_{4}{ }^{3-}$ ) were investigated by FT-IR analysis. Before measurement, the samples were prepared as follows: $\alpha$-Lys or $\varepsilon$ Lys $(12 \mathrm{mg})$ was mixed with $20 \mathrm{~mL}\left(\mathrm{NH}_{4}\right)_{2} \mathrm{HPO}_{4}$ solution $(27$ $\mathrm{mM}$ ) and further stirring for $2 \mathrm{~h}$ at $20^{\circ} \mathrm{C}$. Then, the resulting product was obtained by freeze-drying. Table $\mathrm{S} 1 \dagger$ shows that the secondary structures of pure pLys had higher $\beta$-sheet content and that of the pLys bound with $\mathrm{PO}_{4}{ }^{3-}$ was composed of other structures ( $\alpha$-helix, $\beta$-sheet and $\beta$-turn). In other words, it reveals that the influence of peptide structure for pLys-HAp formation was negligible.

The STEM images, EDX maps and EDX spectra of $\alpha$-pLysHAp (40 mg) and $\varepsilon$-pLys-HAp (40 mg) are displayed in Fig. 3 and S2. $\dagger$ Calcium, phosphorous and nitrogen elements can be assigned to HAp and the peptide. The STEM images showed a homogeneous distribution of nitrogen. It confirmed that the distribution of pLys in pLys-HAp particles was consistent.
(A)

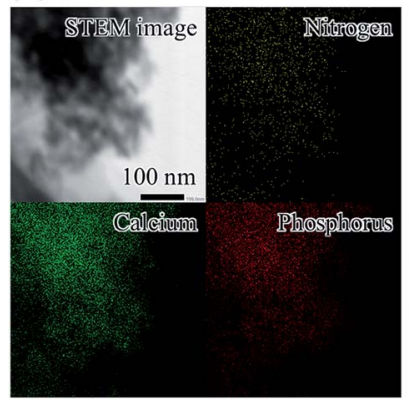

(B)

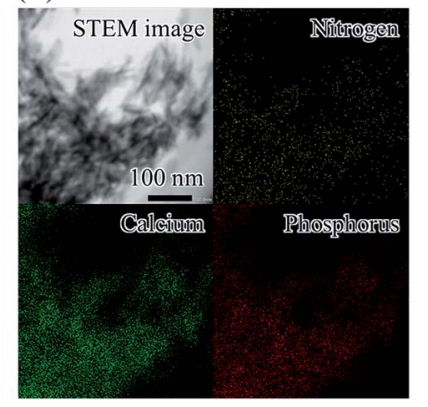

Fig. 3 STEM images and EDX maps of elements of (A) $\alpha$-pLys-HAp (40 $\mathrm{mg}$ ) and (B) $\varepsilon$-pLys-HAp (40 mg). Yellow, green and red colours display nitrogen, calcium and phosphorus elements, respectively.

To confirm the peptide content in composites, TG-DTA analysis was performed and the relative amounts of peptides were calculated from weight losses in the range of $200-700{ }^{\circ} \mathrm{C}$ correspond to the loss of the peptides (Fig. S3† and Table 1). For $\alpha$-pLys-HAp (20, 30 and $40 \mathrm{mg}$ ), the relative amounts of $\alpha$-pLys were 11,11 and $13 \mathrm{mg}$, respectively. On the other hands, the $\varepsilon$ pLys amounts in $\varepsilon$-pLys-HAp (20, 30 and $40 \mathrm{mg}$ ) were $5.5,4.1$ and $4.3 \mathrm{mg}$, respectively. As a result, $\alpha$-pLys amounts included in $\alpha$-pLys-HAp were approximately $13 \mathrm{mg}$ (about $7 \mathrm{wt} \%$ in pLysHAp), whereas $\varepsilon$-pLys-HAp can be included at a maximum of $6 \mathrm{mg}$ (about $3 \mathrm{wt} \%$ in pLys-HAp) for $\varepsilon$-pLys.

The zeta-potential charge of these composites is listed in Table 1. The surface charges of $\alpha$-pLys-HAp with different amounts of $\alpha$-pLys $(20,30$ and $40 \mathrm{mg})$ are $+13.9,+15.4$ and $+14.0 \mathrm{mV}$, respectively, while the surface potentials of $\varepsilon$-pLysHAp $(20,30$ and $40 \mathrm{mg}$ ) are $+4.2,+2.8$ and $+4.2 \mathrm{mV}$, respectively. Taken together, the surface potentials of $\alpha$-pLys-HAp were higher positively charged than that of $\varepsilon$-pLys-HAp. Additionally, 
these results can be attributed to the amount of peptides in pLys-HAp from the TG-DTA data.

\subsection{Effect of the difference in the structure of the peptides on GOX adsorption}

To determine the adsorption mechanism, two common isotherm models (Freundlich and Langmuir models) were taken in this study. ${ }^{52}$ Sun et al. investigated that lysozyme was adsorbed on chitosan/hydroxyapatite hybrid membrane by these models. The equilibrium data were fitted well with Freundlich isotherm models more than Langmuir isotherm models. ${ }^{53}$

The Langmuir isotherm model shows that the GOX adsorption on the surface of pLys-HAp occurs by monolayer adsorption. It is expressed as

$$
Q_{\mathrm{e}}=\frac{Q_{\mathrm{m}} K_{\mathrm{L}} C_{\mathrm{s}}}{1+K_{\mathrm{L}} C_{\mathrm{s}}}
$$

where $C_{\mathrm{s}}$ is equilibrium concentration of GOX in supernatant $\left(\mathrm{mg} \mathrm{mL} \mathrm{m}^{-1}\right), Q_{\mathrm{e}}$ is amount of GOX adsorbed at equilibrium on pLys-HAp (mg mg $\left.{ }^{-1}\right), Q_{m}$ is the maximum capacity of GOX at pLys-HAp (mg mg ${ }^{-1}$ ) and $K_{\mathrm{L}}$ is Langmuir constant $\left(\mathrm{mL} \mathrm{mg}^{-1}\right)$.

In this study, the significance of Freundlich isotherm over the Langmuir isotherm is to confirm the heterogeneous adsorption on these samples. The Freundlich isotherm could be expressed using the following equation:

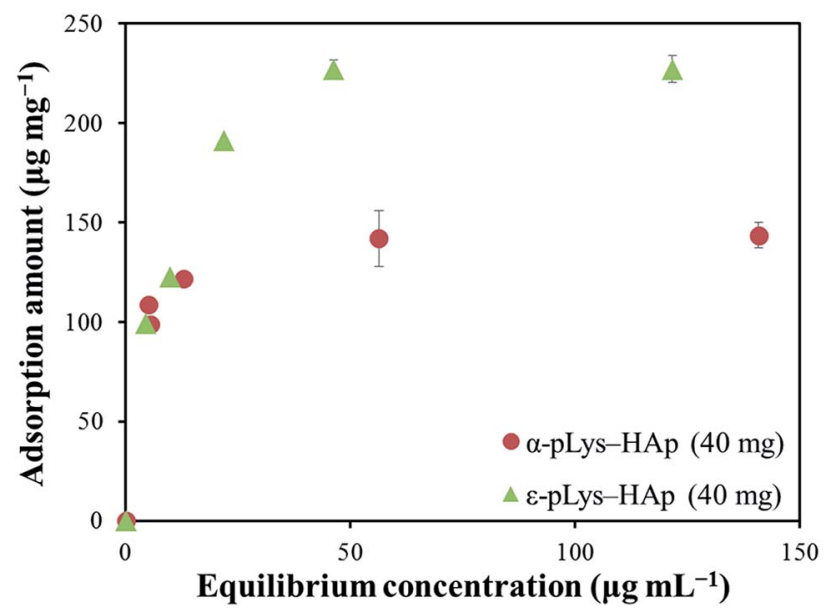

Fig. 4 Adsorption isotherm curves for GOX on $\alpha$-pLys-HAp (40 mg) and $\varepsilon$-pLys-HAp (40 mg).

$$
Q_{\mathrm{e}}=K_{\mathrm{F}} C_{\mathrm{s}}^{\frac{1}{n}}
$$

where $K_{\mathrm{F}}$ is Freundlich constant $\left(\mathrm{ng} \mathrm{\textrm {mg } ^ { - 1 }}\right.$ ) and $1 / n$ is Freundlich adsorption intensity constant. Moreover, ' $n$ ' is reciprocal of slope value for Freundlich systems. It is empirically known that the adsorption behaviour would suggest more heterogeneous systems if the slope is close to $0 .^{54}$

Fig. 4 shows the adsorption isotherm plots for GOX on pLysHAp, and Table 2 presents the isotherm parameters. As can be observed, the acceptable correlation coefficients $\left(R^{2}\right)$ for the Langmuir models were 0.9999 and 0.9974 for $\alpha$-pLys-HAp (40 $\mathrm{mg}$ ) and $\varepsilon$-pLys-HAp (40 mg), respectively. Additionally, the $R^{2}$ values for the Freundlich models attributed to $\alpha$-pLys-HAp (40 $\mathrm{mg}$ ) and $\varepsilon$-pLys-HAp (40 $\mathrm{mg}$ ) were 0.8878 and 0.8795 , respectively. A better value of $R^{2}$ was obtained when Langmuir isotherm models was used. Based on linear regression, it can be considered that the Langmuir model is fitted for adsorption of GOX on $\alpha$-pLys-HAp (40 mg) and $\varepsilon$-pLys-HAp (40 mg). From Fig. 4, the maximum adsorption capacity of GOX according to $\alpha$ pLys-HAp (40 mg) and $\varepsilon$-pLys-HAp (40 mg) were 150 and $230 \mu \mathrm{g}$ $\mathrm{mg}^{-1}$, respectively.

\subsection{Determination of the amino group density in pLys-HAp}

To reveal the difference in the maximum capacity for GOX immobilised on pLys-HAp, the amino group density in pLysHAp composites was evaluated using the fluorescence probe Rhodamine B with isocyanate group. The amino group densities were 16.3 and $15.9 \mathrm{nmol} \mathrm{m}^{-2}$ for $\alpha$-pLys-HAp (40 mg) and $\varepsilon^{-}$ pLys-HAp (40 mg), respectively. According to these results, the densities of amino group in pLys-HAp were not changed between $\alpha$-pLys-HAp (40 mg) and $\varepsilon$-pLys-HAp (40 mg).

From these results, we can discuss about the relationship between the amino group in pLys-HAp and the amount of immobilised GOX on these materials in detail. First, we suppose that all amino groups in these composites cannot be used for enzyme and protein adsorption. Especially, there were relatively many peptides in $\alpha$-pLys-HAp (Fig. S3 $\uparrow$ and Table 1 ), and the longer side chains in $\alpha$-pLys-HAp were easy to move freely in an unsteady manner. Therefore, there is possibility that a part of amino groups formed HAp and adsorbed on the surface of HAp (something like $\mathrm{OH}^{-}$and $\mathrm{PO}_{4}{ }^{3-}$ ). In contrast, it was considered that the amino groups in $\varepsilon$-pLys-HAp should exist on the surface of particles stably. Therefore, the amino groups in $\varepsilon$-pLys-HAp was capable to be efficiently used for enzyme adsorption compared with those in $\alpha$-pLys-HAp. Furthermore, it revealed

Table 2 Adsorption parameters of GOX on $\alpha$-pLys-HAp (40 mg) and $\varepsilon$-pLys-HAp (40 mg) calculated according to Langmuir and Freundlich

\begin{tabular}{|c|c|c|c|c|c|c|}
\hline Sample & \multicolumn{3}{|c|}{ Langmuir parameters } & \multicolumn{3}{|c|}{ Freundlich parameters } \\
\hline$\varepsilon$-pLys-HAp (40 mg) & 0.24 & 31 & 0.9974 & 0.275 & 79.3 & 0.8795 \\
\hline
\end{tabular}
models

${ }^{a} Q_{\mathrm{m}}$ is the maximum adsorption capacity of GOX at equilibrium. ${ }^{b} K_{\mathrm{L}}$ is equilibrium constant for the Langmuir models. ${ }^{c} 1 / n$ is Freundlich adsorption intensity constant, while ' $n$ ' indicates reciprocal of slope value. ${ }^{d} K_{\mathrm{F}}$ is equilibrium constant for the Freundlich models. 
(A)

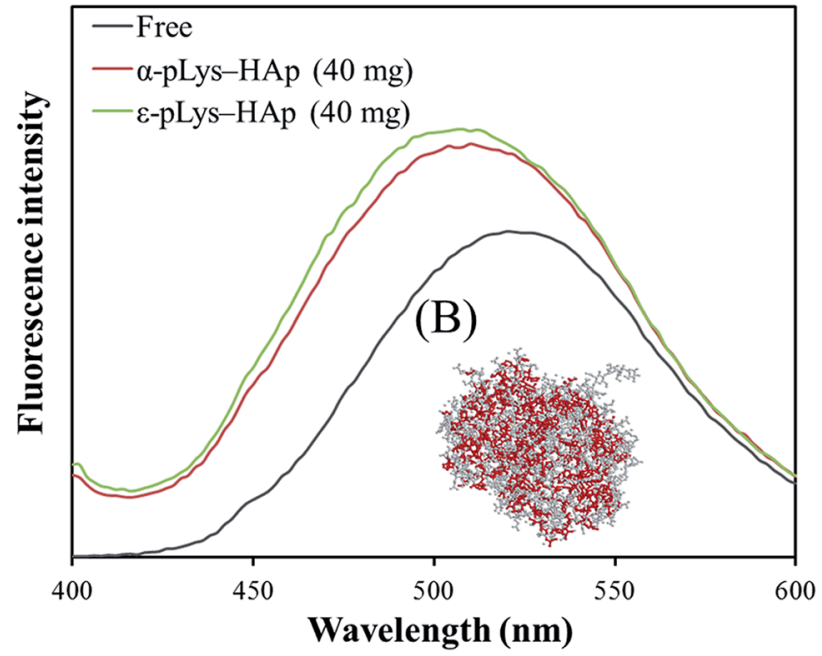

Fig. 5 (A) Fluorescence spectra of free and immobilised GOX. (B) The three-dimensional structure of GOX was obtained from Protein Data Bank; ID: 1CF3. Hydrophobic amino residues in GOX were emphasised using red colour.

that there was not much difference between $\alpha$-pLys-HAp and $\varepsilon$ pLys-HAp for the results of the amino group density calculated by Rhodamine $\mathrm{B}$. The reason is that the same number of amino groups in $\alpha$-pLys-HAp and $\varepsilon$-pLys-HAp may have been utilised for Rhodamine $\mathrm{B}$ adsorption owing to the low molecular weight

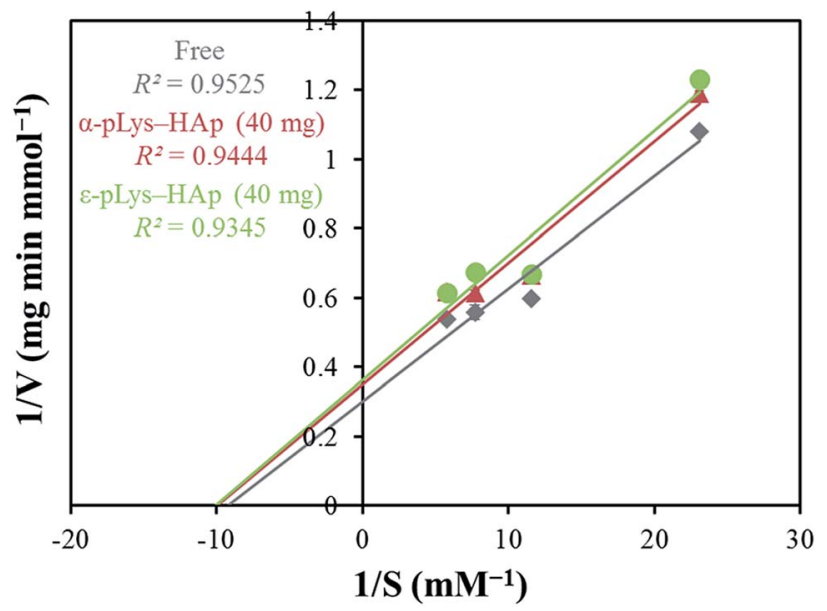

Fig. 6 The Lineweaver-Burk plots of free and immobilised GOX. of Rhodamine B $\left(M_{\mathrm{w}}=536.1\right)$. Nevertheless, in the case of GOX with high $M_{\mathrm{w}}$ of $160000 \mathrm{Da}$, more amino groups used for GOX adsorption on $\alpha$-pLys-HAp because the distance between the amino groups in two pLys molecules in $\alpha$-pLys-HAp is closer than that in $\varepsilon$-pLys-HAp. Thus, it assumed that $\varepsilon$-pLys-HAp had high adsorption capacity of GOX using few amino groups effectively.

\subsection{Analysis of conformational changes in GOX immobilised on pLys-HAp}

8-Anilino-1-naphthalenesulfonic acid (ANS) is an excellent fluorescence probe for binding to the hydrophobic domains of the protein. Therefore, ANS was bound to GOX to investigate the interactions between GOX and pLys-HAp composites. Fig. 5(A) shows the fluorescence spectra of ANS-binding GOX immobilised on $\alpha$-pLys-HAp (40 mg) and $\varepsilon$-pLys-HAp (40 mg). The threedimensional structure of GOX was obtained determined from Protein Data Bank; ID: 1CF3. As Fig. 5(A) shows, the enhancement of the maximum emission intensity in the spectra and its blue-shift were observed for the immobilised GOX bound with ANS. These results indicated that the conformational change in GOX occurred due to the GOX immobilisation on pLys-HAp. ${ }^{37}$

\subsection{Enzymatic kinetics and the catalytic activity of GOX on pLys-HAp}

Fig. S4 $\uparrow$ shows the catalytic activities of GOX (free and immobilised on pLys-HAp). The relative activities of free GOX, immobilised on $\alpha$-pLys-HAp ( $40 \mathrm{mg}$ ) and $\varepsilon$-pLys-HAp ( $40 \mathrm{mg}$ ) are 1.97, 1.57 and $1.59 \mathrm{mmol} \mathrm{min}^{-1} \mathrm{mg}^{-1}$, respectively. Thus, the oxidation activity of GOX immobilised on these materials exhibited more than $80 \%$ compared to that of native GOX (considered to be $100 \%$ ).

Fig. 6 shows the Lineweaver-Burk plot and Table 3 summarises the values of $V_{\max }, K_{\mathrm{m}}, K_{\text {cat }}$ and $K_{\text {cat }} / K_{\mathrm{m}} \cdot V_{\max }$ indicates the ability that how many times enzymes can catalyse a reaction per minute. $V_{\max }$ in the immobilised samples is lower than the native-free one. It can be assumed that these results were caused by the conformational change in GOX immobilised on pLys-HAp (Fig. 5). However, the $K_{\mathrm{m}}$ value of the immobilised GOX is lower than the native GOX, which signified a higher affinity to the substrate. The ratio of $K_{\text {cat }} / K_{\mathrm{m}}$ signifies the catalytic efficiency, and the ratios for GOX immobilised on $\alpha$-pLysHAp (40 mg) and $\varepsilon$-pLys-HAp (40 mg) are slightly lower than free GOX. From these results, GOX immobilised on pLys-HAp samples suggested a high ability for enzyme catalyst.

Table 3 Enzyme catalytic parameters of native or immobilised GOX

\begin{tabular}{|c|c|c|c|c|}
\hline Sample & $\begin{array}{l}V_{\max }^{a} \\
\left(\operatorname{mmol~} \min ^{-1} \mathrm{mg}^{-1}\right)\end{array}$ & $\begin{array}{l}K_{\mathrm{m}}^{b} \\
(\mu \mathrm{M})\end{array}$ & $\begin{array}{l}K_{\text {cat }}{ }^{c} \\
\left(\times 10^{5} \mathrm{~min}^{-1}\right)\end{array}$ & $\begin{array}{l}K_{\mathrm{cat}} / K_{\mathrm{m}}{ }^{d} \\
\left(\times 10^{3} \mu \mathrm{M}^{-1} \mathrm{~min}^{-1}\right)\end{array}$ \\
\hline Free & 3.31 & 108 & 176 & 164 \\
\hline$\varepsilon$-pLys-HAp (40 mg) & 2.75 & 99.2 & 147 & 148 \\
\hline
\end{tabular}

${ }^{a} V_{\max }$ is the maximum velocity. ${ }^{b} K_{\mathrm{m}}$ is Michaelis constant. ${ }^{c} K_{\text {cat }}$ is turn over number. ${ }^{d} K_{\text {cat }} / K_{\mathrm{m}}$ is specificity constant. 


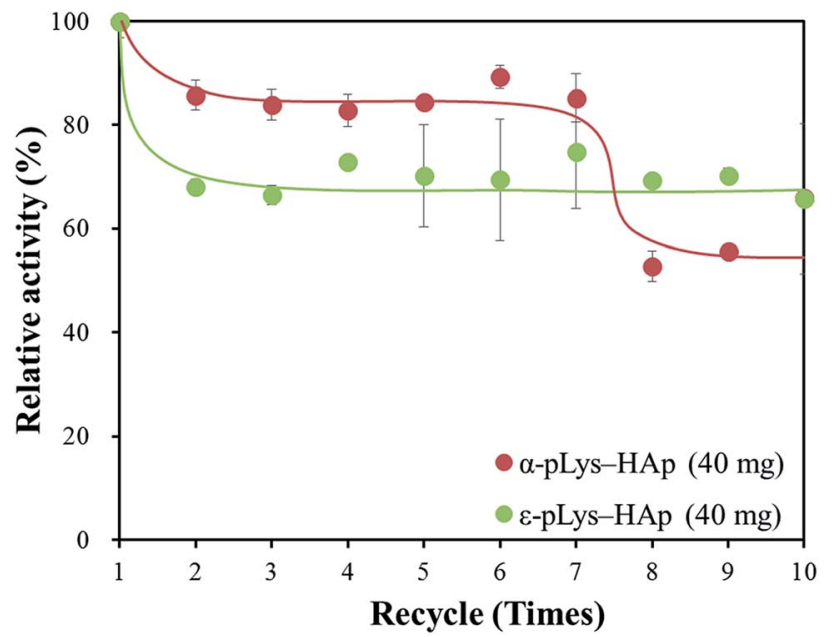

Fig. 7 Remaining activity of GOX immobilised on pLys-HAp in cycling test.

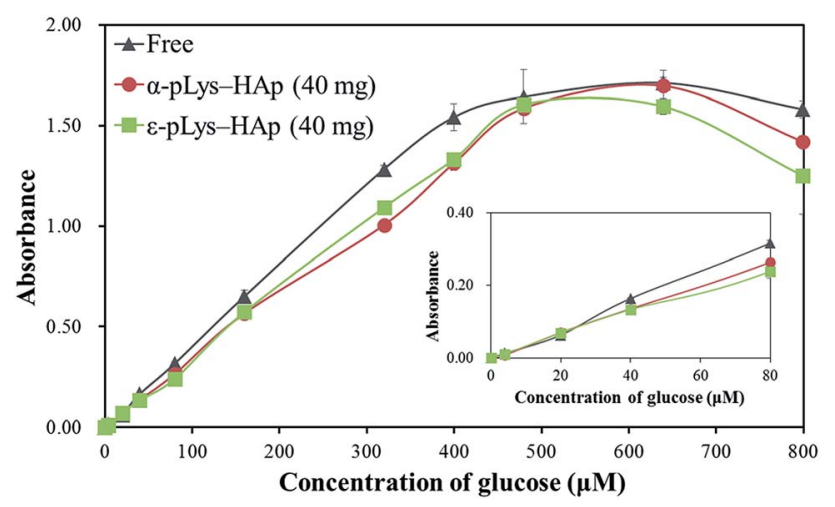

Fig. 8 The glucose-sensitivity using GOX (free and immobilised on pLys-HAp). The inset shows the plot in the range of $4-80 \mu \mathrm{M}$ of glucose.

Moreover, cycling test was carried out and the remaining activity was measured after each cycle. Fig. 7 shows, the relative activity of GOX immobilised on these pLys-HAp materials was kept more than $50 \%$ of the initial activity after ten cycles. For $\alpha$ pLys-HAp (40 mg), the oxidation activity remained about $80 \%$ after seven cycles. On the other hand, the remaining activity of GOX immobilised on $\varepsilon$-pLys-HAp (40 mg) was kept approximately $70 \%$ after ten cycles. These results verified that the pLysHAp composites could be better candidates for immobilising carriers of enzymes with high stability. Additionally, it can be considered that these differences of enzyme stability between $\alpha$ pLys-HAp (40 mg) and $\varepsilon$-pLys-HAp (40 mg) may have been affected by peptide structures and pores in pLys-HAp composites (Fig. S1 $\dagger$ ). First of all, a GOX molecule immobilisation on $\alpha$ pLys-HAp (40 mg) was supported by many amino groups in $\alpha$ pLys and pores with a diameter of $30 \mathrm{~nm}$, although the relative activity was decreased by repeated washing with deionised water after seven cycles. Next, a GOX molecule immobilisation on $\varepsilon$-pLys-HAp (40 mg) was held by few amino groups in $\varepsilon$-pLys, which appeared close to the surface of composites; therefore, the remaining activity was kept high after ten cycles. The difference of the pores in composites and the distance between amino groups in two pLys molecules may become important the key roles for enzyme stability.

To reveal the glucose-sensitivity for GOX immobilised on pLys-HAp particles, these response were measured in the range of glucose concentration of $4-800 \mu \mathrm{M}$, as shown in Fig. 8. In both cases of GOX immobilised on $\alpha$-pLys-HAp (40 mg) and $\varepsilon^{-}$ pLys-HAp (40 mg), the glucose response curves show a linear range from 4 to $400 \mu \mathrm{M}$ of glucose with high correlation coefficients (the $R^{2}$ values were 0.9980 and 0.9987 for $\alpha$-pLys-HAp (40 mg) and $\varepsilon$-pLys-HAp (40 mg), respectively). This range in the immobilised samples was also obtained using GOX solution (native-free) with a high $R^{2}$ value (0.9991). Liu et al. reported the synthesis of graphdiyne (GDY) composites with dual-enzymatic activity via immobilisation of ferrous ion and GOX on GDY sheet. A linear calibration plot between the absorbance at $450 \mathrm{~nm}$ and glucose concentration was shown in the range of 5$160 \mu \mathrm{M}$ of glucose. ${ }^{55}$ It revealed that the as-synthesised $\alpha$-pLysHAp (40 mg) and $\varepsilon$-pLys-HAp (40 mg) could be utilised as glucose sensors with high sensitivity.
(A)

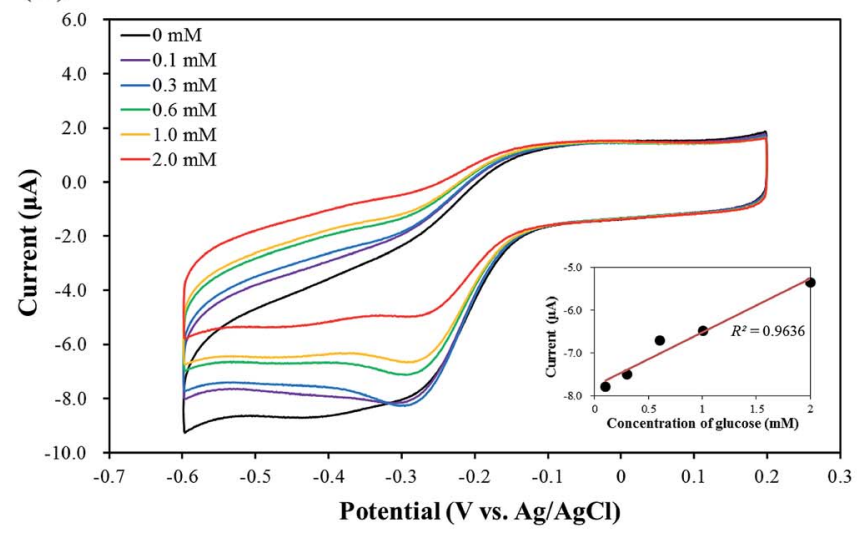

(B)

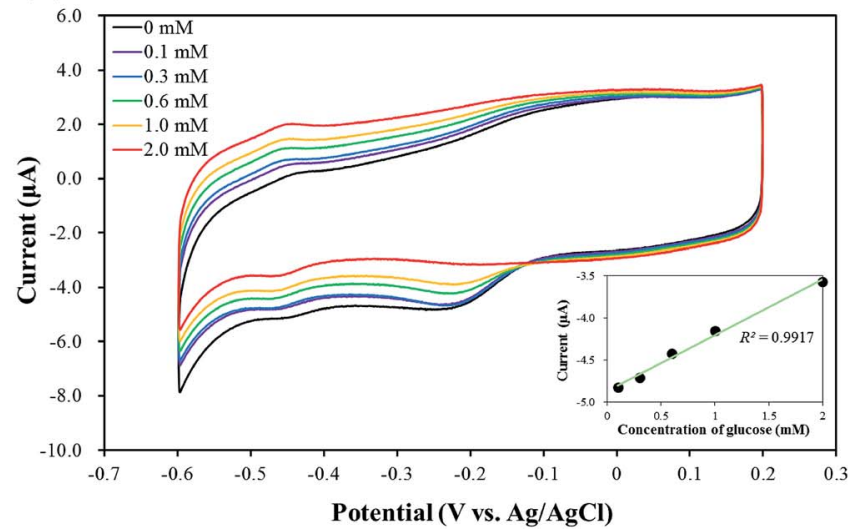

Fig. 9 Cyclic voltammograms of (A) graphene/imogolite/GOX/ $\alpha$-pLys-HAp (40 mg)-modified GCE and (B) graphene/imogolite/GOX/ع-pLysHAp (40 mg)-modified GCE for the addition of 0-2.0 mM glucose in $\mathrm{O}_{2}$-saturated Dulbecco's PBS (pH 7.3) at a scan rate of $100 \mathrm{mV} \mathrm{s} \mathrm{s}^{-1}$. The insets in (A) and (B) show the linear plots for the concentration of glucose vs. peak current. 
3.6 Detecting glucose at the graphene oxide/imogolite/GOX/ pLys-HAp modified GCE

Fig. 9 shows cyclic voltammograms of graphene oxide/ imogolite/GOX/pLys-HAp modified GCE. A voltammetric peak was monitored in the presence of glucose at a potential of $-0.47 \mathrm{~V}$. The reaction could be expressed as follows: $:^{56}$

$$
\begin{gathered}
\text { Glucose }+ \text { GOX }(\text { FAD }) \rightarrow \text { gluconolactone }+ \text { GOX }\left(\text { FADH }_{2}\right) \\
\text { GOX }\left(\text { FADH }_{2}\right)+\mathrm{O}_{2} \rightarrow \text { GOX }(\text { FAD })+\mathrm{H}_{2} \mathrm{O}_{2} \\
\mathrm{O}_{2}+4 \mathrm{H}^{+}+4 \mathrm{e}^{-} \rightarrow \mathrm{GOX}(\text { FAD })+2 \mathrm{H}_{2} \mathrm{O}
\end{gathered}
$$

As Fig. 9(B) shows, a good linear relationship was observed at the range of glucose concentration between 0 and $2.0 \mathrm{mM}$, the current peak at the graphene oxide/imogolite/GOX/E-pLys-HAp (40 mg)/GCE was linearly increased by improving the concentration of glucose (the $R^{2}$ value $\left.=0.9917\right)$. Therefore, the graphene oxide/imogolite/GOX/ع-pLys-HAp (40 mg) modified GCE is a good enzyme electrode and could be used for glucose detection with high sensitivity.

\section{Conclusions}

Two kinds of poly-L-lysine ( $\alpha$-pLys and $\varepsilon$-pLys) containing hydroxyapatite (pLys-HAp) were synthesised via an easy method. The amount of peptide in $\alpha$-pLys-HAp is three times higher than that in $\varepsilon$-pLys-HAp. However, the adsorption capacity of glucose oxidase (GOX) immobilised on $\varepsilon$-pLys-HAp is higher than that on $\alpha$-pLys-HAp. Thus, few amino groups in $\varepsilon$-pLys-HAp could be used for GOX adsorption effectively, while many amino groups in $\alpha$-pLys-HAp were used. The oxidation activities of GOX immobilised on pLys-HAp materials were more than $80 \%$ compared to that of native GOX (considered to be $100 \%$ ). Furthermore, the remaining activity of GOX immobilised on these materials was kept more than $50 \%$ of the initial activity after ten cycles. For $\alpha$-pLys-HAp (40 mg), the oxidation activity remained about $80 \%$ after seven cycles, and the relative activity of GOX immobilised on $\varepsilon$-pLys-HAp (40 mg) was kept approximately $70 \%$ after ten cycles. The difference of the pores in composites and the distance between amino groups in two pLys molecules become important key roles for enzyme stability. GOX immobilised on pLys-HAp particles was able to detect glucose from 4 to $400 \mu \mathrm{M}$. From the results of the direct electrochemistry by cyclic voltammetry (CV) method, the graphene oxide/imogolite/GOX/ $/$-pLys-HAp (40 mg) modified GCE was a good enzyme electrode with high sensitivity for glucose detection. These results suggest that the synthesised particles could be good candidates for immobilising carriers of enzymes with high stability and for biosensors with high sensitivity.

\section{Conflicts of interest}

There are no conflicts to declare.

\section{Acknowledgements}

The author thanks Dr Hiroyuki Iwata, Associate Professor of Aichi Institute of Technology for his help in the STEM-EDX observation. The financial support of Grants-in-Aid for Scientific Research (C) No. 15K06474 from the Japan Society for the Promotion of Science and A-STEP (AS282I011e) from the Japan Science and Technology Agency is gratefully acknowledged.

\section{References}

1 A. Othman, A. Karimi and S. Andreescu, J. Mater. Chem. B, 2016, 4, 7178-7203.

2 M. Sun, S. Huang, L. Chen, Y. Li, X. Yang, Z. Yuan and B. Su, Chem. Soc. Rev., 2016, 45, 3479-3563.

3 C. Anichini, W. Czepa, D. Pakulski, A. Aliprandi, A. Ciesielski and P. Samori, Chem. Soc. Rev., 2018, 47, 4860-4908.

4 F. Demir, B. Demir, E. E. Yalcinkaya, S. Cevik, D. O. Demirkol, U. Anik and S. Timur, RSC Adv., 2014, 4, 50107-50113.

5 X. Cao, Y. Sun, Y. Ye, Y. Li and X. Ge, Anal. Methods, 2014, 6, 1448-1454.

6 A. Kaffash, H. R. Zare and K. Rostami, Anal. Methods, 2018, 10, 2731-2739.

7 L. Wu, X. Lu, X. Wang, Y. Song and J. Chen, Anal. Methods, 2015, 7, 3347-3352.

8 D. Li, Y. Ma, Y. Zhang and Z. Lin, Anal. Methods, 2016, 8, 6231-6235.

9 G. Shtenberg, N. Massad-Ivanir and E. Segal, Analyst, 2015, 140, 4507-4514.

10 H. Liu, C. Tao, Z. Hu, S. Zhang, J. Wang and Y. Zhan, RSC Adv. , 2014, 4, 43624-43629.

11 H. Jang and D. Min, RSC Adv., 2015, 5, 14330-14332.

12 Y. Luo, F. Song, X. Wang and Y. Wang, CrystEngComm, 2017, 19, 2996-3002.

13 J. N. Vranish, M. G. Ancona, S. A. Walper and I. L. Medintz, Langmuir, 2018, 34, 2901-2925.

14 F. Eslamipour and P. Hejazi, RSC Adv., 2016, 6, 20187-20197. 15 M. Abbasi, R. Amiri, A. Bordbar, E. Ranjbakhsh and A. Khosropour, Appl. Surf. Sci., 2016, 364, 752-757.

16 A. T. E. Vilian, S. Chen, M. A. Ali and F. M. A. Al-Hemaid, $R S C$ Adv., 2014, 4, 30358-30367.

17 S. Kumar-Krishnan, M. G. García, E. Prokhorov, M. EstevezGonzález, R. Pérez, R. Esparza and M. Meyyappan, J. Mater. Chem. A, 2017, 5, 7072-7081.

18 A. Paul, G. Vyas, P. Paul and D. N. Srivastava, ACS Appl. Nano Mater., 2018, 1, 3600-3607.

19 Y. Wang, L. Zhang, K. Cui, S. Ge, P. Zhao and J. Yu, ACS Appl. Mater. Interfaces, 2019, 11, 5114-5122.

20 Z. Kang, K. Jiao, R. Peng, Z. Hu and S. Jiao, RSC Adv., 2017, 7, 11872-11879.

21 Y. Chung, Y. Ahn, M. Christwardana, H. Kim and Y. Kwon, Nanoscale, 2016, 8, 9201-9210.

22 C. Li, H. Zhang, P. Wu, Z. Gong, G. Xu and C. Cai, Analyst, 2011, 136, 1116-1123. 
23 G. Bharath, R. Madhu, S. Chen, V. Veeramani, A. Balamurugan, D. Mangalaraj, C. Viswanathan and N. Popandian, J. Mater. Chem. B, 2015, 3, 1360-1370.

24 S. Kojima, F. Nagata, S. Kugimiya and K. Kato, Appl. Surf. Sci., 2018, 458, 438-445.

25 S. Kojima, F. Nagata, M. Inagaki, S. Kugimiya and K. Kato, New J. Chem., 2019, 43, 427-435.

26 K. M. Hawkins, S. S.-S. Wang, D. M. Ford and D. F. Shantz, J. Am. Chem. Soc., 2004, 126, 9112-9119.

27 M. M. Tomczak, D. D. Glawe, L. F. Drummy, C. G. Lawrence, M. O. Stone, C. C. Perry, D. J. Pochan, T. J. Deming and R. R. Naik, J. Am. Chem. Soc., 2005, 127, 12577-12582.

28 Y. Zhu, W. Meng, H. Gao and N. Hanagata, J. Phys. Chem. C, 2011, 115, 13630-13636.

29 S. B. Hartono, W. Gu, F. Kleitz, J. Liu, L. He, A. P. J. Middelberg, C. Yu, G. Q. M. Lu and S. Z. Qiao, ACS Nano, 2012, 6, 2104-2117.

30 J. V. Bradley, L. N. Bridgland, D. E. Colyer, M. J. Duer, T. Friščć, J. R. Gallagher, D. G. Reid, J. N. Skepper and C. M. Trasler, Chem. Mater., 2010, 22, 6109-6116.

31 C. Gao, Y. Wan, X. Lei, J. Qu, T. Yan and K. Dai, Cellulose, 2011, 18, 1555-1561.

32 Y. Ding, J. Liu, X. Jin, H. Lu, G. Shen and R. Yu, Analyst, 2008, 133, 184-190.

33 T. Kaneko, F. Nagata, S. Kugimiya and K. Kato, Ceram. Int., 2018, 44, 20581-20585.

34 D. Sarkar, RSC Adv., 2013, 3, 24389-24399.

35 D. K. Ban and S. Paul, Colloids Surf., B, 2016, 146, 577-584.

36 F. Wang, X. Wang, M. Zhang, A. Huang and L. Ma, Int. J. Biol. Macromol., 2018, 117, 532-537.

37 K. Nakanishi, M. Tomita, H. Nakamura and K. Kato, J. Mater. Chem. B, 2013, 1, 6321-6328.

38 X. Cao, Y. Li, Z. Zhang, J. Yu, J. Qian and S. Liu, Analyst, 2012, 137, 5785-5791.

39 K. Nakanishi, M. Tomita and K. Kato, $R S C A d v ., 2014,4$, 4732-4735.
40 A. T. E. Vilian and S. Chen, RSC Adv., 2014, 4, 50771-50781. 41 L. Wang, Y. Zeng, A. Shen, Y. Fu, L. Zeng and J. Hu, RSC Adv., 2016, 6, 86025-86033.

42 K. Kato, K. Inukai, K. Fujikura and T. Kasuga, New J. Chem., 2014, 38, 3591-3599.

43 V. K. Mishra, B. N. Bhattacharjee, D. Kumar, S. B. Rai and O. Parkash, New J. Chem., 2016, 40, 5432-5441.

44 W. Amer, K. Abdelouahdi, H. R. Ramananarivo, M. Zahouily, A. Fihri, K. Djessas, K. Zahouily, R. S. Varma and A. Solhy, CrystEngComm, 2013, 16, 543-549.

45 W. Fang, H. Zhang, J. Yin, B. Yang, Y. Zhang, J. Li and F. Yao, Cryst. Growth Des., 2016, 16, 1247-1255.

46 X. Zhao, Y. Zhu, F. Chen, B. Lu, C. Qi, J. Zhao and J. Wu, CrystEngComm, 2013, 15, 7926-7935.

47 R. Gonzalez-McQuire, J. Chane-Ching, E. Vignaud, A. Lebugle and S. Mann, J. Mater. Chem. B, 2004, 14, 22772281.

48 V. Humblot, C. Méthivier and C. Pradier, Langmuir, 2006, 22, 3089-3096.

49 Z. Sun, G. Zhang, X. Wang, Z. Gao, X. Cheng, S. Zhang and D. Xu, Cryst. Growth Des., 2009, 9, 3251-3259.

50 V. G. Demillo and X. Zhu, J. Mater. Chem. B, 2015, 3, 83288336.

51 Y. Choi, N. Thongsai, A. Chae, S. Jo, E. B. Kang, P. Paoprasert, S. Y. Park and I. In, J. Ind. Eng. Chem., 2017, 47, 329-335.

52 H. E. Khal and N. H. Batis, New J. Chem., 2015, 39, 35973607.

53 J. Sun, L. Wu and J. Chen, Cellulose, 2016, 23, 3861-3874.

54 K. Y. Foo and B. H. Hameed, Chem. Eng. J., 2010, 156, 2-10.

55 J. Liu, X. Shen, D. Baimanov, L. Wang, Y. Xiao, H. Liu, Y. Li, X. Gao, Y. Zhao and C. Chen, ACS Appl. Mater. Interfaces, 2019, 11, 2647-2654.

56 C. Chen, Q. Xie, D. Yang, H. Xiao, Y. Fu, Y. Tan and S. Yao, RSC Adv., 2013, 3, 4473-4491. 